Jing-fu Liua), Jing-bo Chao'b), Mei-juan Wen ${ }^{\text {b) }}$ Gui-bin Jianga)

a) Research Center for EcoEnvironmental Sciences, Chinese Academy of Sciences, P.O. Box 2871, Beijing 100085, China

b) Department of Chemistry, Beijing University of Science and Technology, Beijing 100083, China

\section{Automatic trace-enrichment of bisphenol A by a novel continuous flow liquid membrane extraction technique}

\begin{abstract}
Bisphenol $A$ was enriched by a novel continuous flow liquid membrane extraction technique, which is based on the combination of continuous flow liquid-liquid extraction and a supported liquid membrane. Related parameters such as flow rates, liquid membrane solvents, and $\mathrm{pH}$ of donor and acceptor were optimized. Using dichloromethane as liquid membrane, over 200 -fold enrichment of $50 \mu \mathrm{g} \mathrm{L}^{-1}$ BPA was obtained after a 40-min enrichment time. The major advantages of this technique are that it provides a relatively high enrichment factor, freedom of choice and long term stability of the liquid membrane, much lower consumption of organic phase, typically 0.05 $\mathrm{mL} \mathrm{min}^{-1}$, scope for full automation, and easy on-line coupling to various detectors.
\end{abstract}

Key Words: Automatic trace-enrichment; Bisphenol A; Water samples; Continuous flow liquid-liquid extraction; Supported liquid membrane

Ms received: June 18, 2001; revised: October 17, 2001; accepted: October 22, 2001

\section{Introduction}

Environmental sources of Bisphenol A (BPA or 4,4'-isopropylidenediphenol) include the thermal degradation of many plastic products and the discharge of BPA manufacturing or processing plants. Recently, much attention has been focused on the compound's estrogenic properties and some authorities have reacted to these concerns by imposing a daily intake limit for BPA. The development of sensitive and selective methods for determination of BPA in environmental and food matrices is a new challenge.

So far, the analysis of BPA has been accomplished mainly by chromatographic methods such as TLC, GC, GC-MS, HPLC, and HPLC-MS. Generally speaking, samples must be pretreated before analysis by these methods. The sample pretreatment procedures applied include Soxhlet extraction [1], liquid-liquid extraction [2, 3], solid-phase extraction (SPE) [4,5], microwave-assisted solvent extraction (MASE) [4], supercritical fluid extraction (SFE) [5], and solid-phase microextraction (SPME) [6]. Among them, the SPE procedure was the most widely used technique.

This study describes an alternative procedure for automatic preconcentration of BPA in water samples. This procedure is based on a new aqueous-organic-aqueous extraction technique that we termed continuous flow liquid membrane extraction (CFLME) [7]. CFLME is the combi-

Correspondence: Prof. Gui-bin Jiang, Research Center for Eco-Environmental Sciences, Chinese Academy of Sciences, P. O. Box 2871, Beijing 100085, China.

E-mail: gbjiang@mail.rcees.ac.cn

Fax: +86 10-62923563 nation of continuous flow liquid-liquid extraction (CFLLE) [8-11] and supported liquid membrane (SLM) extraction [12-14]. In this proposed procedure, BPA was first extracted into the organic phase in the CFLLE step, and then transported onto the organic liquid membrane that formed on the surface of the micro porous membrane of the SLM equipment. Finally, it passes through the liquid membrane and was trapped by the acceptor. Parameters related to CFLME were studied and an enrichment factor of over 200 was obtained after 40 min enrichment of $50 \mu \mathrm{g} \mathrm{L}^{-1} \mathrm{BPA}$. The proposed method is very stable, fully automatic and easy coupled on-line to various detectors.

\section{Experimental}

\subsection{Apparatus}

A schematic diagram of the flow system is shown in Figure 1. It consisted of a FIA 5020 Analyzer (Tecator, Sweden), FIA-3000 flow injection analyzer (SNK, Japan), a manually controlled 6-port valve, and a custom-made SLM device. The mixing coil (MC) and extraction coil (EC) were made of $0.5 \mathrm{~mm}$ ID polytetrafluoroethylene (PTFE) tubing and T-shaped three-way connectors made from PTFE were used to mix solutions or solvents.

The custom-made SLM device is the same as described elsewhere [7]. Briefly, a sheet of Fluoropore FG PTFE porous membrane (Millipore Corp., Bedford, MA.) was clamped between two custom-made PTFE blocks with grooves of $0.5 \mathrm{~mm}$ deep, $2.0 \mathrm{~mm}$ wide, and $50 \mathrm{~mm}$ long to form two channels (the acceptor channel and the donor channel) with the same volume of $50 \mu \mathrm{L}$. 


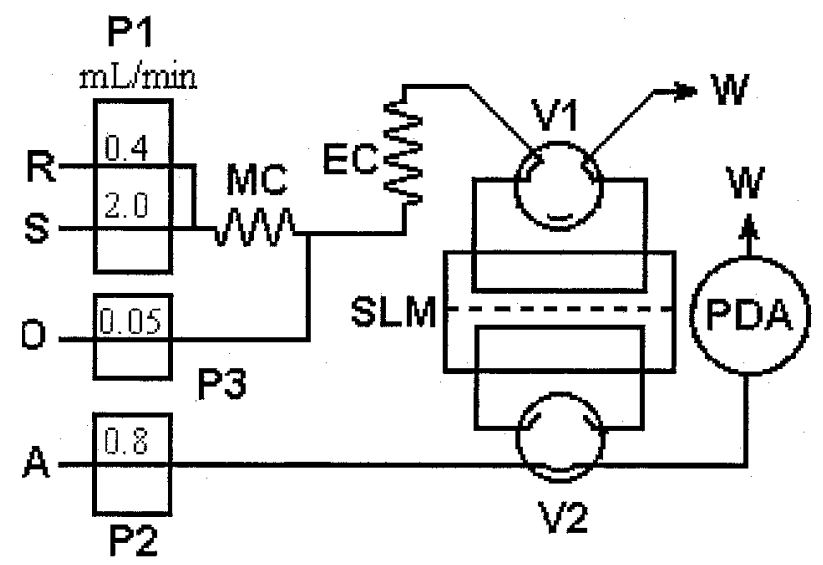

Figure 1. Schematic diagram of the proposed continuous flow liquid membrane extraction system manifold. See text for details.

An SPD-M10Avp diode array detector (Shimadzu, Japan) setting at $240 \mathrm{~nm}$ wavelength was used as detector. Data acquisition and process were accomplished with a ClassVP Workstation (Shimadzu, Japan), which was connected with the detector with an SCL-10Avp system controller (Shimadzu, Japan).

\subsection{Reagents}

Bisphenol A (BPA, 97\%) was purchased from Acros Organics (NJ, USA). Standard stock solution was prepared by dissolving $20 \mathrm{mg}$ of standard in $100 \mathrm{~mL}$ of water and stored at $4{ }^{\circ} \mathrm{C}$. Working solutions were obtained daily by appropriate dilution of the stock solutions with water. All other chemicals were purchased from Beijing Chemicals Corporation and were analytical grade or better. Deionized water was used throughout.

\subsection{Procedure}

A schematic diagram of the proposed system is shown in Figure 1 and the installation of the whole system was described in our previous paper [7].

As shown in Figure 1, sample solution (S) and diluted sulfuric acid $(R)$ are introduced into the system by a peristaltic pump (P1) of the FIA 5020 Analyzer. These two solutions are mixed in a mixing coil (MC) and are segmented by organic solvent $(\mathrm{O})$ delivered by the piston pump (P3) of the FIA-3000 Flow injection analyzer. BPA is first extracted into the organic phase in an extraction coil (EC). Then, by switching $\mathrm{V} 1$ at the sampling position, the segments of aqueous solution and organic solvent are introduced into the donor side of the SLM to form an organic liquid membrane on the surface of the micro porous membrane. The acceptor (A) stream, delivered by a peristaltic pump (P2) of the FIA 5020 Analyzer, is kept stagnant in the acceptor channel of SLM by switching V2 at the bypass position. BPA therefore diffuses through the liquid membrane and is trapped by the alkaline acceptor. After a certain time (typically $20 \mathrm{~min}$ ), $\mathrm{V} 1$ is switched to the bypass position and V2 is switched to the injection position to introduce BPA that has been enriched in the acceptor channel of the SLM to the detector (PDA), and the peak height is determined.

All the solutions were degassed by ultrasound for $30 \mathrm{~min}$ before use to prevent the formation of air bubbles in the flow system.

\section{Results and discussion}

\subsection{Influence of the CFLLE related parameters}

It is well known that the inner diameter and length of extraction coil, the flow rates of sample, reagent, and organic solvent, and the ratio of aqueous to organic phase significantly influence the extraction efficiency of a CFLLE system. In this study, optimization of these parameters was conducted by 20 -min enrichment of $50 \mu \mathrm{g} \mathrm{L}^{-1}$ BPA. A solution of $0.32 \mathrm{M} \mathrm{H}_{2} \mathrm{SO}_{4}$ was used to acidify the donor and a solution of $12 \mathrm{~g} \mathrm{~L}^{-1} \mathrm{Na}_{2} \mathrm{HPO}_{4} \cdot 12 \mathrm{H}_{2} \mathrm{O}$ was used as acceptor.

Experiments show that the mixing coil $(E C)$ did not affect the peak height in the range of $30-100 \mathrm{~cm}$. A $30-\mathrm{cm}$ long mixing coil was therefore used throughout.

The length and inner diameter of the extraction coil has a significant influence on the peak height. As small tubing diameter is helpful for extraction, $0.5 \mathrm{~mm}$ ID PTFE coil was adopted without optimization, while the coil length was optimized in the range of $60-360 \mathrm{~cm}$. Results show that the greatest peak height was obtained when a 240$\mathrm{cm}$ long extraction coil was used. Therefore, a $240-\mathrm{cm}$ long extraction coil was adopted.

To decrease the dilution factor of sample in the flow system, the flow rate of sulfuric acid should be as low as pos-

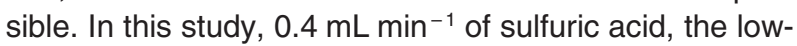
est flow rate that can be obtained, was used throughout. The adopted flow rate of acceptor, which does not affect the extraction efficiency, was $0.8 \mathrm{~mL} \mathrm{~min}^{-1}$.

Keeping the sample flow rate at $1.2 \mathrm{~mL} \mathrm{~min}^{-1}$, the flow rate of organic phase, dichloromethane, was optimized in the range of $0.05-0.20 \mathrm{~mL} \mathrm{~min}^{-1}$ and the results show that the peak height decreased with increasing flow rate of dichloromethane, as expected. Though it might be helpful for attaining a greater peak height, a lower flow rate than

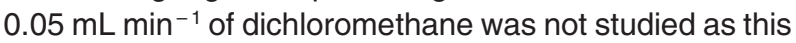
is the lowest flow rate the instrument can provided. Therefore, $0.05 \mathrm{~mL} \mathrm{~min}^{-1}$ was used in the following studies. 




Figure 2. Effect of solvents on the peak heights of $50 \mu \mathrm{g} \mathrm{L}^{-1}$ BPA. Enrichment time, 20 min; acceptor, $12 \mathrm{~g} \mathrm{~L}^{-1} \mathrm{Na}_{2} \mathrm{HPO}_{4}$. $12 \mathrm{H}_{2} \mathrm{O}$; samples were acidified with $0.5 \mathrm{M} \mathrm{H}_{2} \mathrm{SO}_{4}$.

Keeping the flow rate of $\mathrm{H}_{2} \mathrm{SO}_{4}$ at $0.4 \mathrm{~mL} \mathrm{~min}^{-1}$ and the concentration of $\mathrm{H}_{2} \mathrm{SO}_{4}$ at $0.08 \mathrm{M}$ after mixing with different flow rates of sample solutions, by using appropriate concentration of $\mathrm{H}_{2} \mathrm{SO}_{4}$, the influence of sample flow rate was investigated from 0.4 to $2.8 \mathrm{~mL} \mathrm{~min}^{-1}$. The results demonstrated that the peak height increased with increasing sample flow rate. Though the highest peak height was obtained at $2.8 \mathrm{~mL} \mathrm{~min}-1,2.0 \mathrm{~mL} \mathrm{~min}^{-1}$ sample flow rate was adopted as the whole flow system is more stable at this flow rate.

\subsection{Solvent used as the liquid membrane}

As BPA is a relatively polar compound, polar solvents should be beneficial for high enrichment rate according to the rule of "like dissolve in like". Three kinds of polar solvents were tested in this study and the results are shown in Figure 2, which indicates that the largest peak height was obtained when dichloromethane was used. Dichloromethane was adopted in the following studies.

\subsection{Sulfuric acid concentration in the donor stream}

BPA is a weak acid, which should be protonated to form an uncharged substance before extraction into organic phase. Sulfuric acid was adopted to acidify the sample solution, and its concentration was optimized by introducing different concentrations of sulfuric acid into the donor stream while keeping the flow rate at $0.4 \mathrm{~mL} \mathrm{~min}^{-1}$. As shown in Figure 3, the greatest peak height was obtained when 0.4-0.8 M sulfuric acid was used. Therefore, $0.5 \mathrm{M}$ sulfuric acid was introduced in the following studies. After

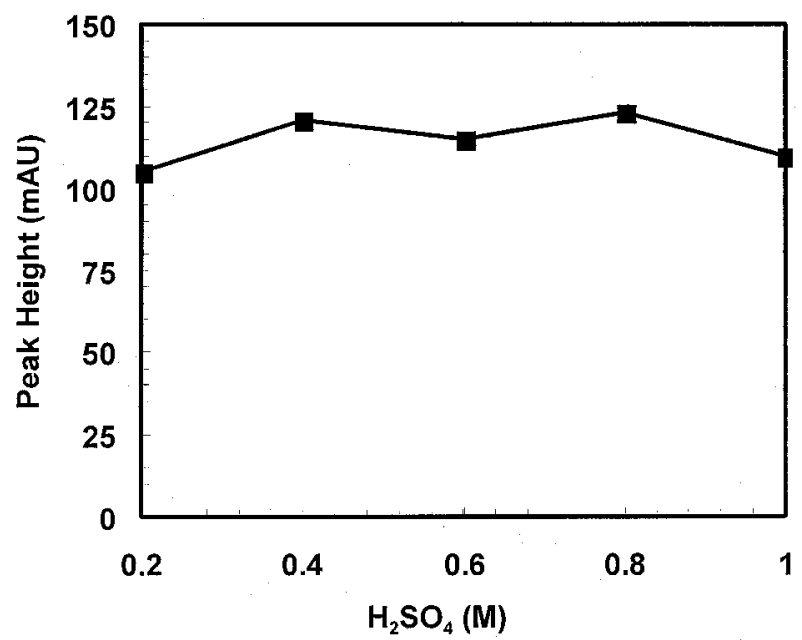

Figure 3. Effect of sulfuric acid concentration on the peak heights of $50 \mu \mathrm{g} \mathrm{L}^{-1}$ BPA. Donor: $2.0 \mathrm{~mL} \mathrm{~min}^{-1}$ of sample solution mixed with $0.4 \mathrm{~mL} \mathrm{~min}^{-1}$ of $\mathrm{H}_{2} \mathrm{SO}_{4}$, and 0.05

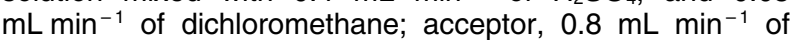
$12 \mathrm{~g} \mathrm{~L}^{-1} \mathrm{Na}_{2} \mathrm{HPO}_{4} \cdot 12 \mathrm{H}_{2} \mathrm{O}$; Enrichment time, $20 \mathrm{~min}$.

mixing with the sample solution in the donor stream, this corresponds to $0.08 \mathrm{M} \mathrm{H}_{2} \mathrm{SO}_{4}$.

\subsection{Effect of the acceptor pH}

The final step of the proposed CFLME procedure is back extraction of BPA into the acceptor solution. In order to trap BPA in the acceptor, an alkaline acceptor should be used to ionize the BPA molecule, which diffuses through the membrane into the acceptor, to prevent it from reentering the liquid membrane. The $\mathrm{pH}$ of acceptor was optimized using Kolthoff buffer solutions $\left(\mathrm{KH}_{2} \mathrm{PO}_{4}-\right.$ $\mathrm{Na}_{2} \mathrm{~B}_{4} \mathrm{O}_{7}$ buffer for $\mathrm{pH} 7.0-9.0, \mathrm{Na}_{2} \mathrm{HPO}_{4}-\mathrm{NaOH}$ buffer for $\mathrm{pH} 10.0-12.0$ ) and $0.1 \mathrm{M} \mathrm{NaOH}$ (for $\mathrm{pH} 13.0$ ). The results shown in Figure 4 demonstrate that the highest peak was obtained at above $\mathrm{pH} 12.0$. This result is in agreement with the theory reported by Jönsson et al. [15], i.e., the optimum $\mathrm{pH}$ should be $\mathrm{pH}>\mathrm{p} K_{\mathrm{a}}+3.3=9.5+3.3$ (the $\mathrm{p}_{\mathrm{a}}$ value of BPA is around 9.5). A $0.1 \mathrm{M} \mathrm{Na}_{2} \mathrm{HPO}_{4}-$ $\mathrm{NaOH}$ buffer solution of $\mathrm{pH} 12.0$ was adopted in the following studies.

\subsection{Influence of salt effect}

It is well known that the addition of salt can usually increase the extraction efficiency. To examine the effect of the addition of salt, different amounts of sodium chloride were added into the sample solution to give $0,5,10,15$, and $20 \% \mathrm{NaCl}$ in the donor after mixing with sulfuric acid solution. The results shown in Figure $\mathbf{5}$ indicate that the peak height of BPA increased significantly up to $15 \%$ $\mathrm{NaCl}$ and then remained unchanged. Therefore, 15\% $\mathrm{NaCl}$ was adopted in the following studies. 


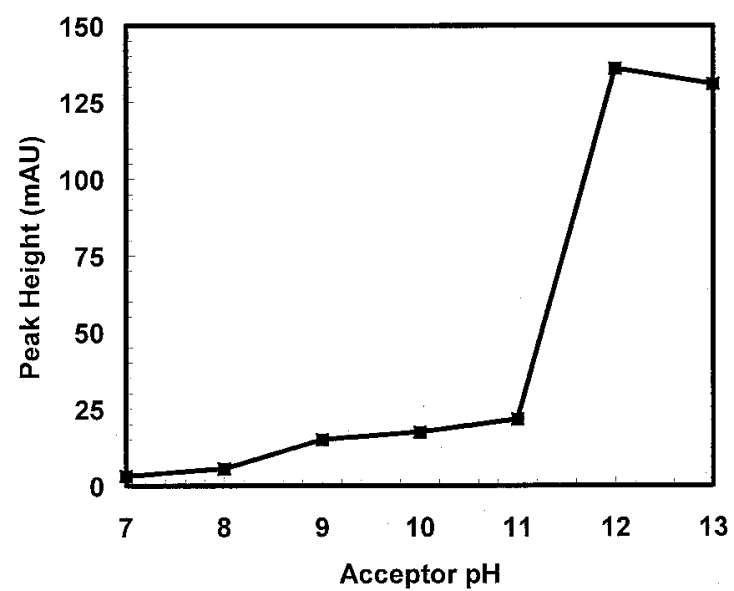

Figure 4. Effect of acceptor $\mathrm{pH}$ on the peak heights of 50

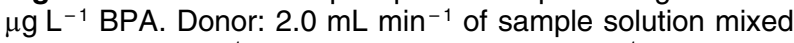
with $0.4 \mathrm{~mL} \mathrm{~min}^{-1}$ of $\mathrm{H}_{2} \mathrm{SO}_{4}$, and $0.05 \mathrm{~mL} \mathrm{~min}^{-1}$ of dichloromethane; acceptor, $0.8 \mathrm{~mL} \mathrm{~min}^{-1}$ of buffer with different $\mathrm{pH}$; Enrichment time, $20 \mathrm{~min}$.

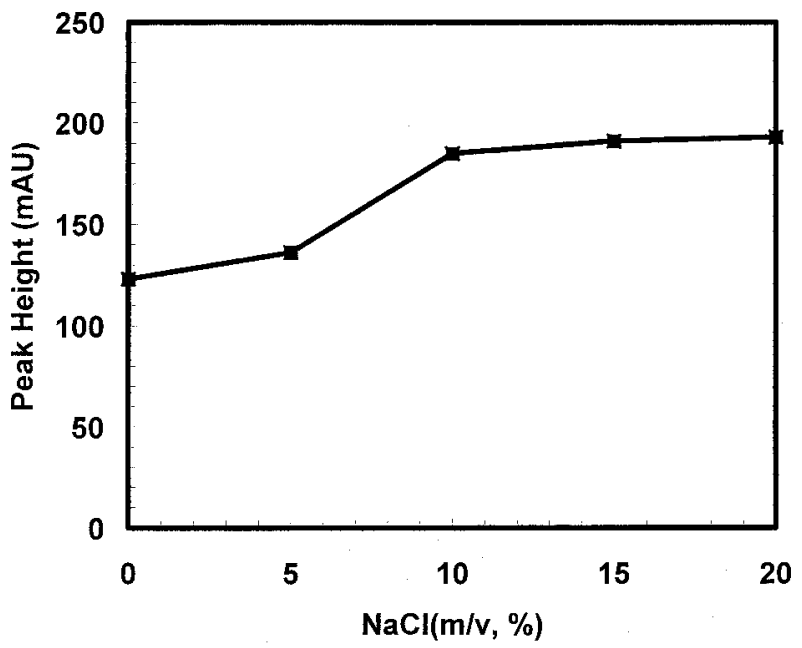

Figure 5. Effect of $\mathrm{NaCl}$ concentration on the peak heights

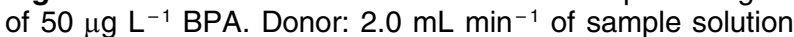
mixed with $0.4 \mathrm{~mL} \mathrm{~min}{ }^{-1}$ of $\mathrm{H}_{2} \mathrm{SO}_{4}$, and $0.05 \mathrm{~mL} \mathrm{~min}^{-1}$ of dichloromethane; acceptor, $0.8 \mathrm{~mL} \mathrm{~min}{ }^{-1}$ of $0.1 \mathrm{M} \mathrm{Na}_{2} \mathrm{HPO}_{4^{-}}$ $\mathrm{NaOH}$ (pH12.0) buffer; Enrichment time, 20 min.

\subsection{Repeatability and linearity}

To examine the repeatability of the system, $50 \mu \mathrm{g} \mathrm{L}^{-1}$ BPA was enriched 5 times with an enrichment time of 20 min. The RSD was $3.4 \%$.

The linearity of the system was investigated by performing 30 min enrichment of a series solutions contains 10,50, 100 , and $200 \mu \mathrm{g} \mathrm{L}^{-1} \mathrm{BPA}$, respectively. Experiments show that the peak height $(A, \mathrm{mAU})$ and concentration $(C$, $\mu \mathrm{g} \mathrm{L}^{-1}$ ) were related by the regression equation $A=4.95 C-16.1$ with a correlation coefficient of $r=0.9986$.

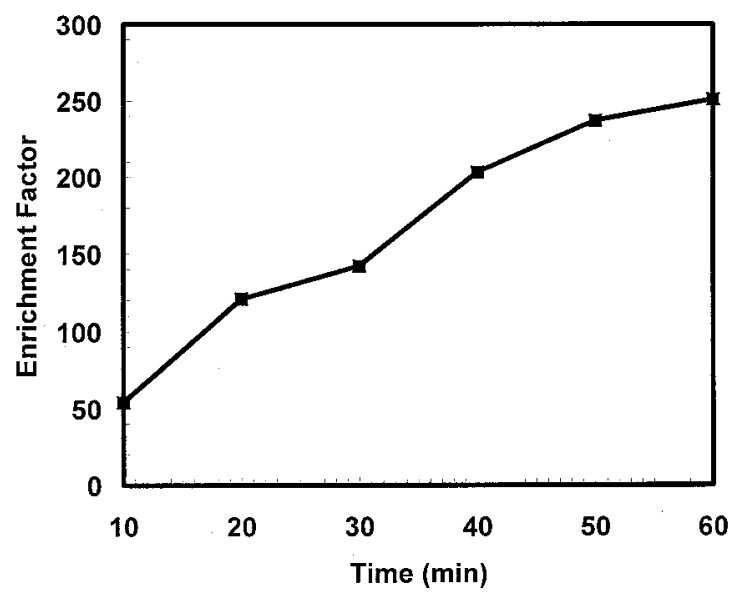

Figure 6. Effect of enrichment time on the enrichment factors of $50 \mu \mathrm{g} \mathrm{L}^{-1}$ BPA. Donor: $2.0 \mathrm{~mL} \mathrm{~min}^{-1}$ of sample solution mixed with $0.4 \mathrm{~mL} \mathrm{~min}^{-1}$ of $\mathrm{H}_{2} \mathrm{SO}_{4}$, and $0.05 \mathrm{~mL} \mathrm{~min}^{-1}$ of dichloromethane; acceptor, $0.8 \mathrm{~mL} \mathrm{~min}{ }^{-1}$ of $0.1 \mathrm{M} \mathrm{Na}_{2} \mathrm{HPO}_{4}^{-}$ $\mathrm{NaOH}$ buffer $(\mathrm{pH} 12.0)$.

\subsection{Influence of enrichment time}

During long term enrichment, the $\mathrm{pH}$ or buffer capacity of the acceptor chamber might change and thus affect the enrichment efficiency. Thus it is necessary to investigate the influence of enrichment time on the enrichment factor.

The enrichment factor was obtained according to

$$
E_{\mathrm{e}}=\left(H_{\mathrm{e}} / C_{\mathrm{e}}\right) /\left(H_{\mathrm{i}} / C_{\mathrm{i}}\right)
$$

Here, $H_{\mathrm{e}}$ is the peak height determined by enriching BPA with initial concentration of $C_{\mathrm{e}} . H_{\mathrm{i}}$ is the peak height obtained by direct injection of a standard BPA solution with concentration of $C_{\mathrm{i}}$ prepared in acceptor. Direct injection was conducted by replacing the SLM unit with a sampling loop of $50 \mu \mathrm{L}$, the same volume of the acceptor channel of the SLM device, while keeping the other conditions the same as in the enrichment process. A $1 \mathrm{mg} \mathrm{L}^{-1} \mathrm{BPA}$ solution was directly injected as the peak height of direction of $50 \mu \mathrm{g} \mathrm{L}^{-1}$ BPA was too low for quantification.

Figure 6 shows the effect of enrichment time in the range of 10-60 min, which indicates that over 200-fold concentration was obtained when samples were concentrated for 40 min or longer.

\section{Conclusion}

Combination of continuous flow liquid-liquid extraction and supported liquid membrane, a novel automatable technique, was developed for trace enrichment of BPA with an enrichment factor of over 200 after $40 \mathrm{~min}$ of enrichment. 
The proposed method has some of the advantages of SLM, such as high enrichment factor, convenient automation, and easy on-line coupling to analytical instruments. Furthermore, this proposed CFLME technique has some advantages over the SLM technique. As polar organic solvents can be used as liquid membrane, this CFLME technique provides freedom of choice of liquid membrane and much higher enrichment rate than that of the original SLM. Another advantage of this CFLME procedure is the robustness of the liquid membrane, which is stable without the danger of the membrane breakthrough, such as often occurred in the original SLM procedure. This procedure needs an additional pump to deliver organic phase and the consumption of organic phase, typically $0.05 \mathrm{~mL} \mathrm{~min}{ }^{-1}$, is a little more than in the original SLM procedure but much less than in continuous flow liquid-liquid extraction.

This proposed CFLME technique is a promising sample pretreatment platform for various selective detectors. Further studies on the on-line coupling of CFLME with HPLC and CE for determination of BPA were conducted in this laboratory.

\section{Acknowledgements}

This work was supported by the National Natural Science Foundation of China (20177026 and 29825114) and the Chinese Academy of Sciences.

\section{References}

[1] J.S. Khim, K. Kannan, D.L. Villeneuve, C.H. Koh, J.P. Giesy, Environ. Sci. Technol. 1999, 33, 4199-4205.

[2] M. del Olmo, A. Gonzalez-Casado, N.A. Navas, J.L. Vilchez, Anal. Chim. Acta 1997, 346, 87-92.

[3] P. Varelis, D. Balafas, J. Chromatogr. A 2000, 883, $163-170$.

[4] S.N. Pedersen, C. Lindholst, J. Chromatogr. A 1999, 864, 17-24.

[5] H. Lee, T.E. Peart, J. AOAC Int. 2000, 83, 290-297.

[6] J. Salafranca, R. Batlle, C. Nerin, J. Chromatogr. A 1999, 864, 137-144.

[7] J. Liu, J. Chao, G. Jiang, Anal. Chim. Acta, submitted.

[8] V. Wallace, Anal. Biochem. 1967, 20, 411-418.

[9] N.R. Kuzel, J. Pharm. Sci. 1968, 57, 852-855.

[10] B. Karlberg, S. Thelander, Anal. Chim. Acta 1978, 98, $1-7$.

[11] F.H. Berggamin, J.X. Medeiros, B.F. Ries, E.A.G. Zagatto, Anal. Chim. Acta 1978, 101, 9-16.

[12] J.Å. Jönsson, L. Mathiasson, Trends Anal. Chem., $1999,18,318-324$.

[13] G. Audunsson, Anal. Chem. 1986, 58, 2714-2723.

[14] J.Å. Jönsson, L. Mathiasson, J. Chromatogr. A 2000, 902, 205-225.

[15] J.Å. Jönsson, P. Lovkvist, G. Audunsson, G. Nilve, Anal. Chim. Acta 1993, 277, 9-24. 